\title{
Analysis of Human Interactive Accounting Management Information Systems Based on Artificial Intelligence
}

\author{
Jin Qiu, Guangdong University of Science and Technology, China*
}

\begin{abstract}
With the gradual improvement of market economy, people' s consumption level is constantly improving, and the quality requirements are getting higher and higher. In order to study the management accounting information analysis platform based on artificial intelligence (AI) and realize the goal of accounting computerization, the application of AI in expert systems is applied to the field of accounting information analysis. The combination of subsystems is applied to the construction of $\mathrm{AI}$ accounting information web system, and the feasibility analysis of its theory and technology is carried out. The results show that its effect is obvious: accelerating the flow of all information and promoting the change of enterprise management mode. Moreover, compared with the traditional system algorithm, the accuracy of the system model is improved by $6 \%$, and the time delay is reduced by $9 \mathrm{~ms}$, which makes the overall management level of the enterprise further improved, the scope of enterprise competition further expanded, and the cost of enterprise saved.
\end{abstract}

\section{KEYWORDS}

Accounting Management Information System, Artificial Intelligence, Expert System, Human Interactive, Web System

\section{INTRODUCTION}

Under the background of the great development of science and technology, how to get the advantageous information quickly from various kinds of data and information with various types and densities is the influencing factor for modern enterprises to gain market competitive advantage and market initiative position (Yang et al., 2020; Shen\&Han, 2020). Audit is a crucial part of the company. If modern enterprises use the traditional way to deal with the massive information and complex data types generated in operation, their work efficiency will be greatly limited and they may face the crisis of enterprise annexation. Therefore, in the modern dynamic change of information, how to improve efficiency and ensure the accuracy of audit information is particularly important (Luo et al., 2018), which has also become the research focus in relevant fields.

With the progress of big data, the rapid acquisition of accounting information and knowledge has become an important resource for enterprises. Modern enterprises in developed countries are striving to become intelligent enterprises, and they have taken knowledge creation value as their goal (Lu et al., 2018). As the development of information technology, China has clearly put forward the sustainable development strategy, information strategy and scientific development strategy in the 
national economic development strategy (André et al., 2018). How to use information technology to improve the management ability and enhance the enterprise competitiveness in the dynamic and complex environment of "information economy" and "knowledge economy" has become a major practical problem for every Chinese enterprise. As an interdisciplinary subject of computing science and management science, data mining has become a crucial part of knowledge acquisition and business intelligence with the development of information technology (Bai et al., 2021; Balthazar et al., 2018; Jiang et al.(2017). The popularity and application of the Internet has led to a sharp increase in the amount of information and data, which provides rich information resources and convenient interactive communication platform for decision support, and enables more experts to participate in decision-making. However, data workers pay little attention to the effective use of data mining to acquire knowledge and provide more assistant decision-making information for enterprise decisionmakers (Kibria et al., 2018). Therefore, how to transform the data and information of enterprises into information resources to obtain sustainable competitive advantage has become a hot topic in academia and business circles.

To sum up, to further improve the economic benefits of enterprises, integrating artificial intelligence (AI) technologies such as big data information processing into accounting information management is of great significance to the economic development and scale expansion of modern enterprises. Therefore, the research innovation is to combine a decision support system with an expert system to design an intelligent decision support system, interactive accounting information system (IAIS). Moreover, the accounting management information model based on IAIS is constructed by adding accounting information and audit knowledge. Finally, its performance and feasibility are analyzed to provide a powerful reference for the development of accounting information in China.

\section{RELATED WORK}

In modern society, keeping pace with the times is the trend of accounting development to adapt to economic development. Kokina and Davenport (2017) studied the impact of AI on accounting and auditing (Kokina \& Davenport, 2017). They explored the potential effects and biases of technology implementation in the current industry and their impact on future research trends by collecting events in the industry. Meanwhile, Marshall and Lambert (2018) proposed that management accounting as indicator decision-making can be regarded as a non-linear system (Marshall\&Lambert, 2018). Decision-making is one of the most important parameters in management accounting, which requires correct information analysis. The AI-based information has the functions of learning, forecasting, classification, and expansion. It can be used as a solution and an auxiliary tool of management accounting. As a branch of AI, the artificial neural network is a qualitative method that can be used to analyze complex information. It has great potential to solve problems that cannot be solved with logic analysis techniques in standard software.

It is found that the use of soft computing technologies such as artificial neural networks can improve the efficiency of information systems. Marshall and Lambert (2018) discussed a framework to support cognitive task automation based on AI technology, and developed a cloud-based intelligent accounting application program by using this framework. The virtual case of sequential design provides a reference for people to understand future accounting intelligence (Wu\&Wu, 2019). Wu et al. (2019) explored the relationship between the positive work emotions of marketing executives and employees' innovative behaviours, analyzed the impact of emotional contagion on innovative behaviours, investigated the mediating role of work engagement and apparent behaviour, and tested the hypothesis with structural equation modelling. The results show that employees' positive emotions positively affect employee's work engagement and innovative behaviour (Richards et al., 2019). Cheng et al. (2021) proposed a new q-rung orthopair fuzzy weighted averaging operator (q-ROFWAO) to rank and evaluate small and medium-sized manufacturing enterprises. The results show that this method is effective in the Sustainability Enterprise Risk Management (SERM) of small and medium-sized 
enterprises (Cheng et al., 2021). Aiming at the static mapping problem in the existing enterprise knowledge map, Yang and Liao (2021) introduced the time dimension to describe the dynamic, sudden and timely evolution characteristics of enterprise risk events. ResNet dynamic knowledge reasoning method was proposed to improve the loss balance function of multi network model. Experiments show that the new model can effectively improve the accuracy of entity and relationship prediction (Yang\& Liao, 2021; Kong et al., 2020; Lv\&Wu, 2021).

Given the above analysis, AI technology plays a crucial role in feature extraction and management analysis of data information. There is massive data information in the process of enterprise audit and accounting management. Expert system, an artificial intelligence technology, is combined with a decision support system to design an intelligent accounting management information system, which is of great practical significance to the progress and intelligent development of modern society.

\section{THEORY AND TECHNOLOGY}

\section{Management Accounting Information Theory}

Different understandings of the nature of accounting determine how a person or an organization uses the functions of the accounting system to achieve its goals. Although both management accounting information and financial accounting information are provided by enterprise management authorities, the former mainly serves the internal management of enterprises. The producers and users of accounting information are unified. There is no need for society to regulate management accounting information and financial accounting information. Management accounting information is single, that is, it can only be used in specific enterprise units, not social. This is the basic principle that management accounting principles cannot be paid attention to by society, but it cannot say that management accounting has no accounting principles. If financial accounting principles have the characteristics of unity, compulsion and legality, management accounting principles have the characteristics of singleness, selectivity, and consciousness (Liu et al., 2021; Liu et al., 2020; Ni et al., 2020). Therefore, management accounting information is not only an artificial system. It can be designed and constructed according to people's wishes, and it also can complete the statistical analysis of data.

A system is usually defined as an organic whole with a specific function, which is connected in a certain structural form and composed of several elements. It includes four concepts: system, element, structure, and function. It shows the relationship between elements and elements, between elements and systems, and between systems and environment. These relations are usually explained by the viewpoint of system theory (Rikhardsson\&Yigitbasioglu, 2018; Ding et al., 2020; Zhou et al., 2019). Based on this point, with the progress of science and technology, people should use system concepts not only for the study and recognization of complex management systems, but also for the activity in various fields such as operation, finance, planning, remuneration, and performance. Only by understanding management from the perspective of system integrity, can people correctly evaluate various management activities and formulate appropriate and reasonable countermeasures for various problems in management, so as to improve the management ability of the organization and create a new situation of efficient management (Dimitrov, 2019; Yi, 2021; Chen\& Sivakumar, 2021).

\section{Al Technology Theory}

AI is to study the law of human intelligence activities, construct an artificial system with certain intelligence, and study how to make the computer complete the work that needed human intelligence to be competent in the past.

One of the aims of AI research is to enable machines to compete for complex tasks that usually require human intelligence. For example, heavy scientific and engineering calculations are originally undertaken by the human brain. Now, the computer can not only calculate, but calculate faster and more accurately than the human brain. The definition of complex work changes with the development 
of the times and the progress of technology, and the specific goal of AI also naturally develops with the changes of the times.

The relationship between AI and thinking science is the relationship between practice and theory. AI is an applied branch of thinking science, which lies in the application level of thinking science technology. From the aspect of thinking, besides logical thinking, AI needs to consider image thinking and inspiration thinking to promote the breakthrough development of AI. Mathematics is often regarded as the basic science of multiple disciplines and it also enters the field of language and thinking. It plays a role in the scope of standard logic and fuzzy mathematics. AI disciplines must use mathematical tools, and they will promote each other and develop faster (Kotb et al., 2019; Yan et al., 2020; Zhang et al., 2020). Fig. 1 shows a wide range of AI-related research areas.

Figure 1. The research field of $\mathrm{Al}$

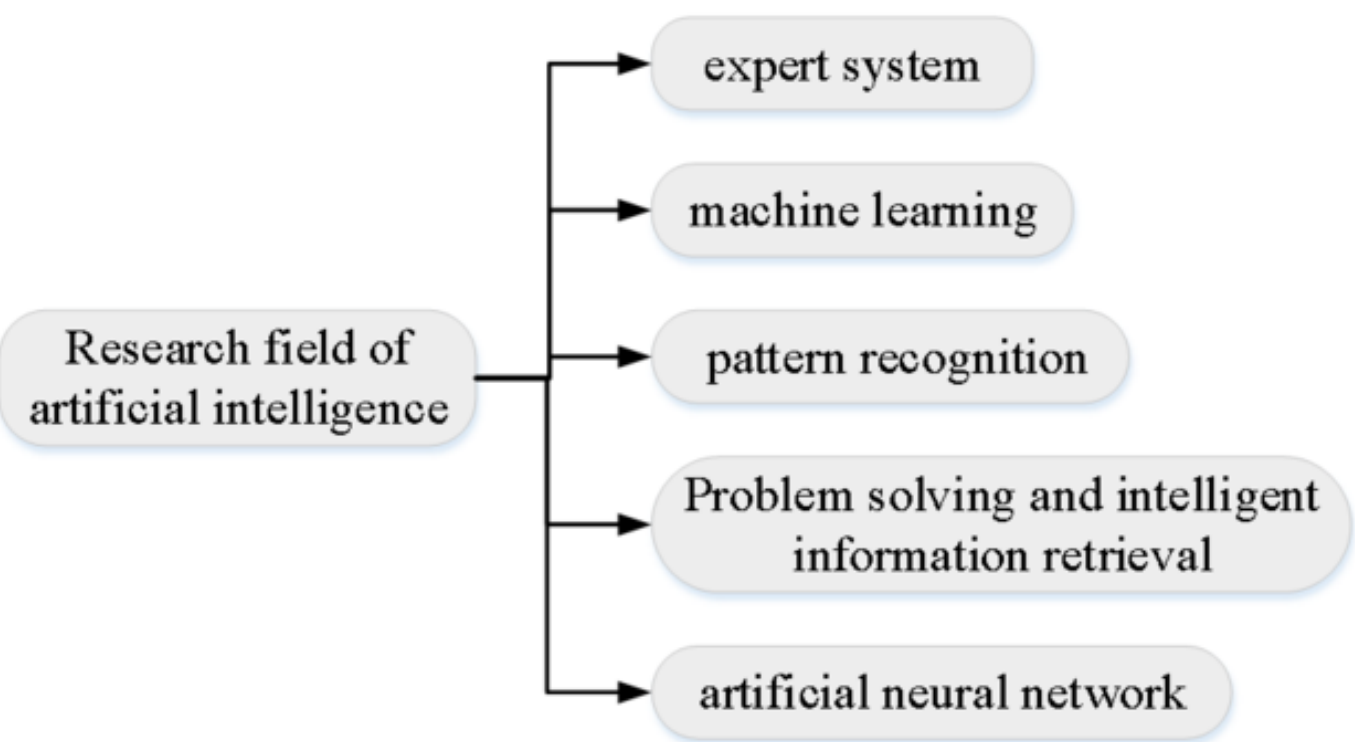

\section{METHOD}

\section{Application of $\mathrm{Al}$ in Management Accounting Information}

The accounting information system under the condition of information technology is comprehensive and open. It is based on the network and is used to analyze, design and establish the accounting business generated by the value movement during enterprise operation. It can provide financial information, assist in financial analysis, prediction and planning, and strengthen financial control and financial decision-making (Yuan \& Wu, 2020; Wu et al., 2020; Deng et al., 2021). However, the current accounting information processing system has obvious deficiencies, so AI technology and network technology are introduced into it. The intelligent professional judgment function in the accounting system is introduced, the intelligent decision support function of accounting information processing in the decision support system is strengthened, and the network interconnection system of the accounting information system is constructed to form a new intelligent and open system (Chen et al., 2020; Shen et al., 2019). The released accounting information system (IAIS) makes it possible 
to share accounting information in a timely and reliable manner, and the function of the accounting information system has been greatly improved.

The wide application of AI shows the inevitability of its application in the field of financial accounting. Today, as the main application of statistical methods in the operation of finance and accounting, AI is a very meaningful research topic. For example, the function of the expert system is to let the computer simulate the expertise, reasoning method and ability of experts in a certain field to solve unquantifiable and complex problems. Therefore, the expert system is an information system working at the expert level. Its application in the accounting information system and the establishment of the computer-aided system can make financial management and decision-making intelligent. This is an effective way to realize the transformation of the accounting information systems from accounting to management and decision-making. The accounting information system under the condition of AI should be an application system that combines humans, computer systems, network systems, data and programs. It has a friendly interface, including both an accounting system and a higher-level accounting decision support system.

The current situation of the Chinese accounting information system has some outstanding shortcomings, such as the simulation of manual accounting system and the weak decision support function. Enterprise finance and accounting cannot meet the increasingly difficult and demanding decision-making problems. Therefore, only by intensifying the development efforts, introducing advanced technology and methods into the accounting information system, and developing highperformance and high-level accounting information system as soon as possible, can people meet the needs of the development of accounting information in China and make the accounting information system play a real core role in enterprise management informatization.

\section{Construction of Accounting Information System Based on Al}

At present, the accounting of accounting information is the difficulty of the intelligent judgment of computer programs, which also becomes the key to restricting the development of accounting information to a higher level. Accounting work has the following procedures. First, the original vouchers of economic business turn to the initial confirmation and measurement of the preparation of accounting vouchers. Second, the registration of accounting vouchers needs to be reconfirmed. Third, the preparation of accounting statements such as accounting books also needs to be reconfirmed. Fourth, the preparation of transfer vouchers at the end of the period needs reconfirmation and measurement, such as cost accounting and depreciation of fixed assets. Finally, accounting confirmation and measurement are still needed when book value is reset. In a word, accounting judgment runs through the activities of accounting confirmation, reconfirmation, and measurement. The research purpose is to introduce intelligent professional judgment into the accounting process and build an intelligent accounting system in IAIS. Fig. 2 is an AI accounting information system.

This system model is based on database technology and AI technology. It consists of two subsystems: a structured accounting system and an unstructured reasoning system. Enterprise economic business is automatically processed by the accounting system, and the relevant accounting information is stored in the financial accounting database, which provides a standard and satisfying data source for the automatic generation of accounting statements.

\section{Al Decision Support System for Accounting Information}

The intelligent decision support system of IAIS is the combination of a model-based decision support system and an expert system in AI. Its core idea is to introduce knowledge representation and knowledge processing in AI into the accounting decision support system. Model-based decision support system mainly carries out quantitative analysis of decision-making problems. With the addition of a knowledge base and its management system and inference engine, the system can make a qualitative analysis of decision-making by using experts' knowledge and experience in solving problems, thus 


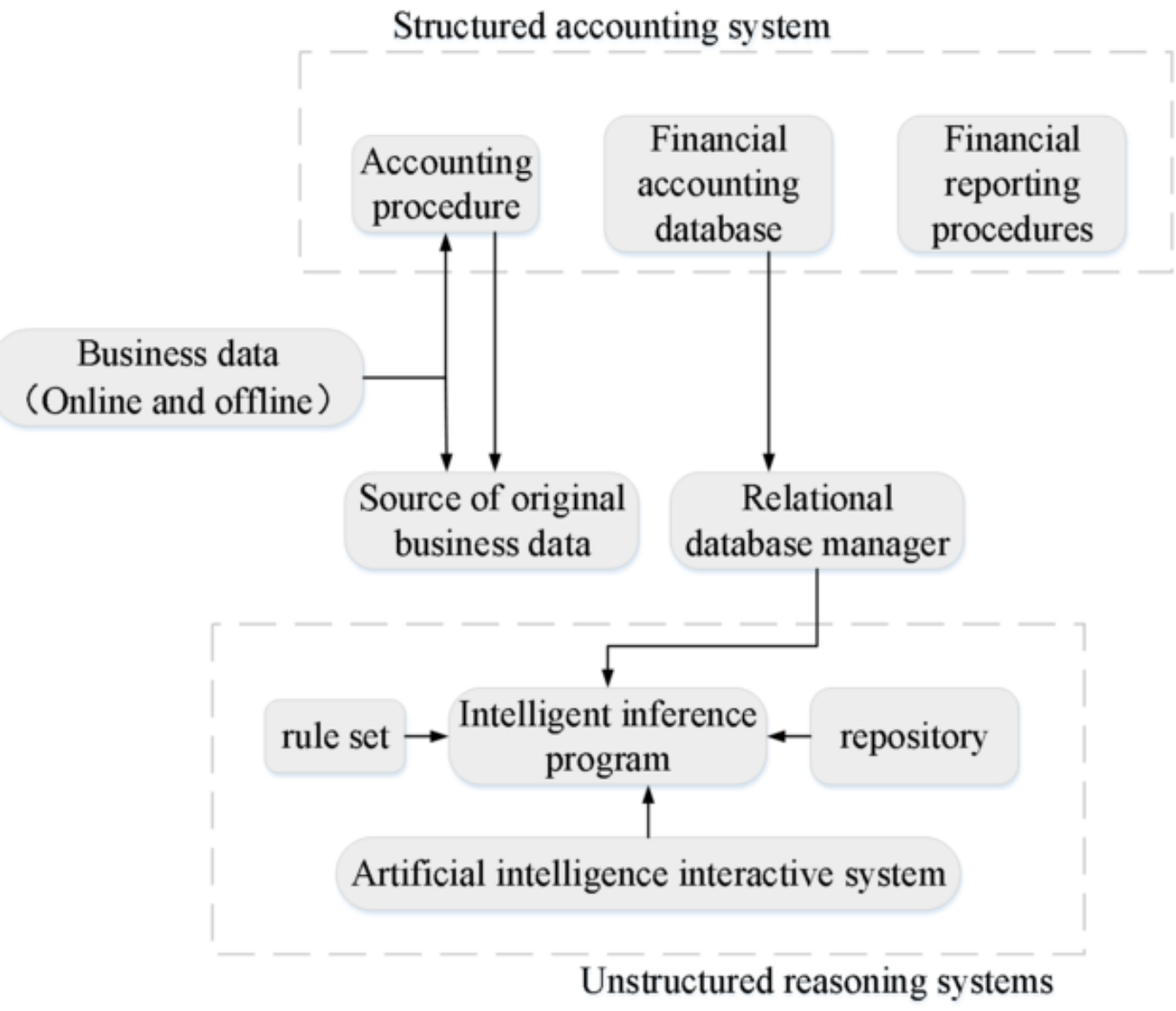

Figure 3. Information prediction processing flow

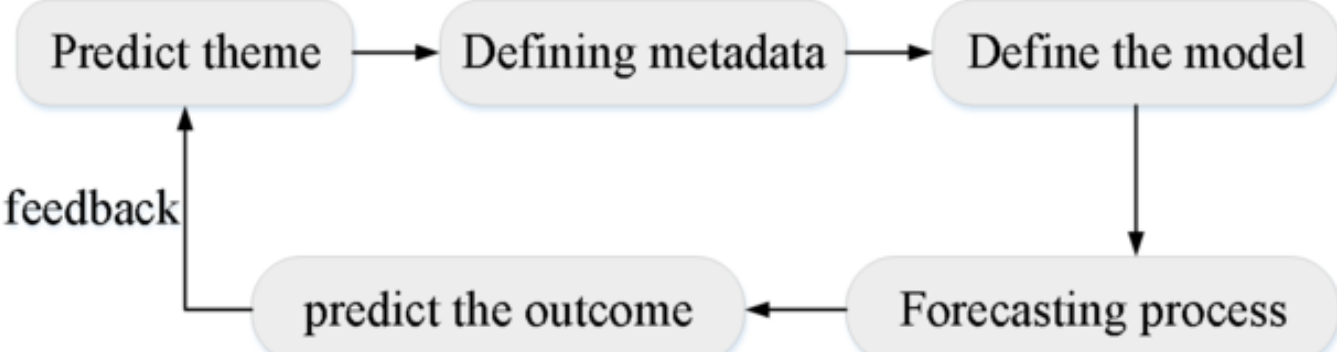


supporting unstructured and semi-structured decision-making. Fig. 3 is the information processing flow of the financial subsystem constructed.

\section{Construction of Intelligent Accounting Information Internet System}

Compared with the traditional internal management and closed and isolated accounting information system, it has become a fatal disadvantage as the competitive environment of enterprises becomes increasingly complex. It is of great significance to enable enterprises to grasp market information in time, grasp the direction of enterprise development, and remain invincible in business competition. This study constructs an accounting information system, which links financial accounting information with other departments inside the enterprise and relevant information outside the enterprise, and

Figure 4. The sketch of accounting information Internet system

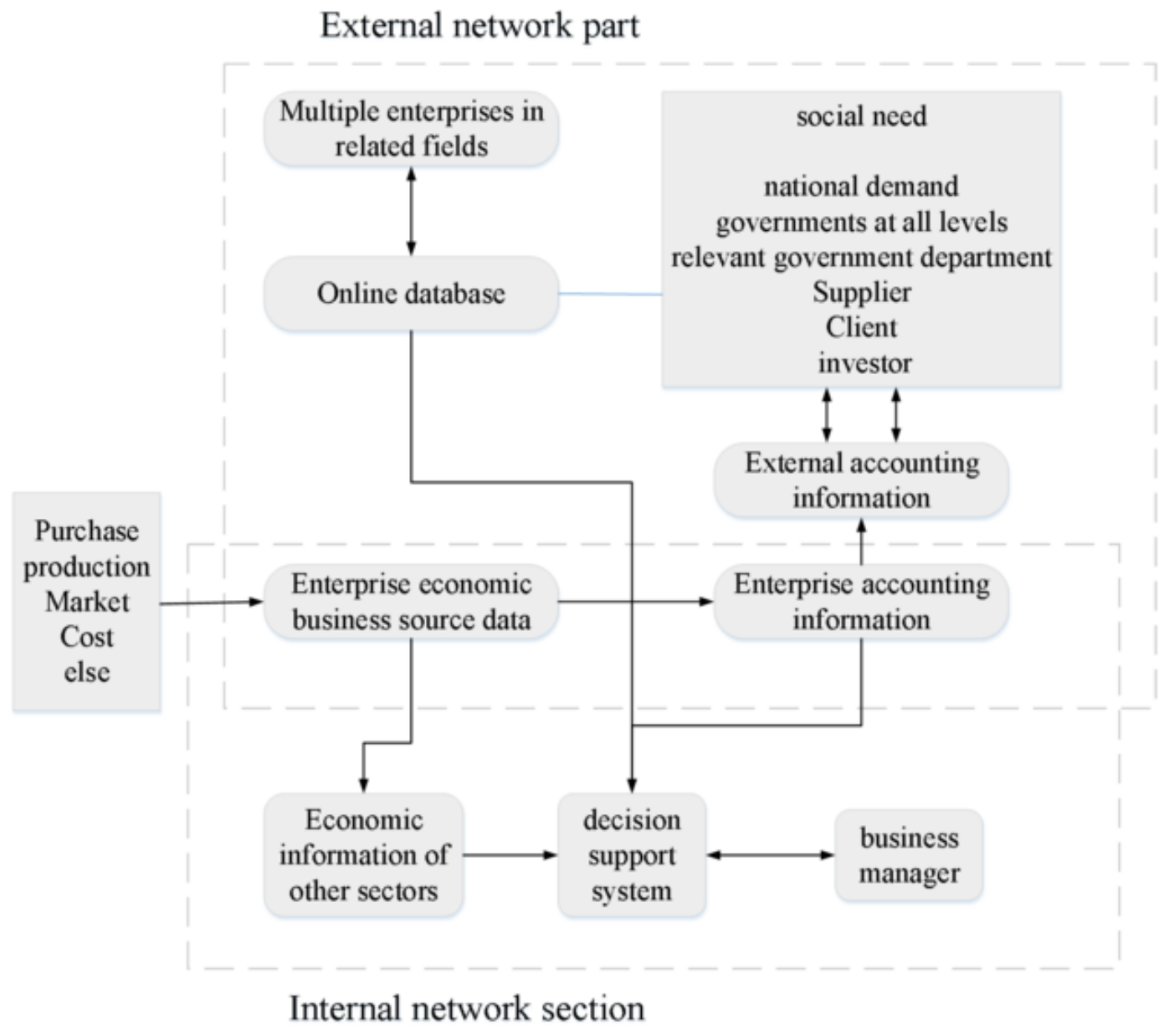


forms a seamless link to the information of each department inside and highly open to the outside. Fig. 4 is architecture design.

After the computer collects the data of the original economic activities of the enterprise, the intelligent accounting information system automatically processes the daily accounts according to the original information collected from the daily business activities of the enterprise, such as filling out vouchers, journals, detailed accounts, and ledgers. Ultimately, it generates the accounting statements needed by enterprises and transmits the internal accounting information after sorting out and processing to the intelligent decision support system. Meanwhile, through the internal and external network interface, IAIS will transfer the economic data useful for managers' decision-making to the enterprise intelligent decision support system. After obtaining three aspects of data, the intelligent decision support system (IDSS) makes a comprehensive analysis and evaluation of these data and the pending decision-making problems, and draws a proposal scheme to provide decision-making support for managers of enterprises.

Enterprises can also publish their own financial information through the Internet. Users of external accounting information authorized and registered by enterprises can easily get useful accounting information for their own decision-making to meet the needs of users of external accounting information. Building a network interconnection system for IAIS makes accounting information sharing and real-time become reality, and makes IAIS more intelligent.

The AI management accounting information-related system constructed is analyzed theoretically and technically through arithmetic. The accuracy and time delay of the system constructed and the traditional management accounting information analysis platform are analyzed.

The theoretical feasibility analysis of this paper will be based on the core logical structure of the theoretical basis of intelligent information processing. The following deduction and analysis are made for the core logic structure of the theoretical basis of intelligent information processing. Physical information $I$ is the integral of ordinal scalar or scalar $r$ :

$$
I=\int \eta(R) d r
$$

where $\eta(R)$ is information density. The information determined by equation (1) is objective information. The real effective information $J$ is the value revision of information $I: J=I^{V}$.

The value coefficient of information is $V \in[0,1]$. When $V=1, J=1$. All information is valuable. When $V=0, J=1$, which only means that there is information. The larger the $V$ is, the more effective the information is. The message is $I$, and what is useful is $J$, which is information. Knowledge $K$ is the integral of effective information $J$.

$$
K=\int \delta I^{V} d I
$$

where $\delta$ is the knowledge conversion coefficient of information. Knowledge determined by equation (2) is objective knowledge. Equation (2) also shows that when the information is regulated, the corresponding knowledge will be affected. On the contrary, knowledge can also be controlled by controlling information. 


\section{RESULTS AND DISCUSSION}

\section{Theoretical Feasibility Analysis}

According to the above theoretical structure, intelligent information processing is to judge and predict the knowledge value of subjective information $\mathrm{J}$ through the comprehensive analysis of input objective information $I$ based on known subject knowledge, and automatically provide results or decision-making suggestions. This is a knowledge innovation process based on $K$, input $I$, parse $J$, and then promote $K^{\prime}$ 's creation (obtaining $K^{\prime}$ ). Expert system is a knowledge system based on the theory of intelligent information processing. IAIS system is based on the model of expert system, which combines AI technology, network technology and traditional accounting information system to form an intelligent accounting information system with the functions of intelligent accounting, intelligent decision support and network interconnection. It should be said that the successful application of an expert system provides strong theoretical support for the construction of IAIS.

\section{Performance Analysis of Management Accounting Information Analysis System Based on Al}

The model constructed is compared with the traditional management accounting information analysis system. Fig. 5 shows the accuracy and time delay analysis.

Figure 5. The curve of performance comparison with traditional system model

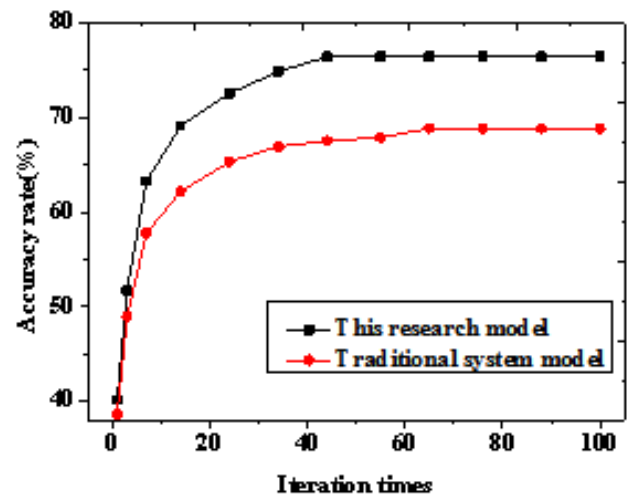

(a) Accuracy

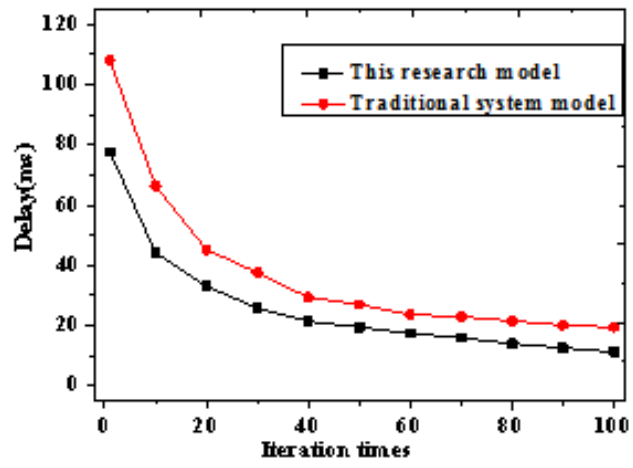

(b) Time delay

The accuracy and time delay of the system model constructed are compared with those of the traditional system model. Compared with the traditional system model, the accuracy of the proposed model is higher. When the number of iterations is 100 , its accuracy rate can reach $76 \%$, while the accuracy of the traditional system model is less than $70 \%$; when the number of iterations is 100 , the time delay of the system model proposed is $11.17 \mathrm{~ms}$ and that of the traditional system model is $19.42 \mathrm{~ms}$. Therefore, from the theoretical point of view, the accuracy and time delay of the proposed system model based on AI are obviously better than those of the traditional system.

\section{Technical Feasibility Analysis}

Traditional accounting information processing is constrained by the ability of manual operation, so the intelligent processing of accounting information can not be realized. However, nowadays, 
the emergence of technologies such as AI, E-commerce, data warehouse, remote communication technology, and Internet/Intranet network technology provides strong technical support for intelligent accounting information system, and makes it possible to establish the IAIS system with powerful information processing functions.

AI technology makes it possible for the traditional accounting information system to become more intelligent. The following three aspects are essential for an intelligent information processing system. First, the knowledge base. It means that the knowledge in the field of analysis is systematically ordered and organized into the machine-identifiable system according to the specific structure. It can be expressed through the semantic network or ontology knowledge tree structure. Its function is to support the knowledge of intelligent information analysis. Second, the ruleset. It means that the analysis strategy is expressed in the form of rules, which means "if... then..." can be used. The function is to standardize the process of analysis, so that the system can analyze according to rules and the content of the knowledge base. Finally, the inference engine is designed based on the knowledge base and ruleset. Its function is to analyze and judge input information according to knowledge and rules, and output decision-making suggestions.

\section{CONCLUSION}

Based on the goal of realizing intelligent enterprise management system, this paper takes enterprise management system as the object, and applies the expert system technology in artificial intelligence to the field of accounting information analysis. The IAIS information decision support system is designed, and the artificial intelligence accounting information system model is further constructed. Finally, the performance of the system is analyzed from the perspective of theoretical and technical feasibility. The results show that the system has made a breakthrough in theory and technology, and the accuracy and delay of the system have been significantly improved, in which the accuracy has been improved by $6 \%$ and the delay is only $11.17 \mathrm{~ms}$. The system will play a greater role in the accounting work and management level of subsequent artificial intelligence of society and enterprises. However, there are still some deficiencies in the research. For example, artificial intelligence is still in the development stage, and its application in accounting information analysis is only a model, which has not been put into practice. Therefore, with the development of science and technology and the maturity of objective conditions, the research on intelligent accounting information system and its related problems will be continuously improved to promote the rapid development of information technology in the whole society.

\section{Conflict of Interest}

None to report.

\section{Funding}

This research was supported by Guangdong Provincial Philosophy and Social Sciences "13th FiveYear Plan" 2020 Disciplinary Co-construction Project (Grant no. GD20XGL54), with the project name of "Research on the Application of the Three-Level Model of Environmental Cost Control for Manufacturing Enterprises in the Guangdong-Hong Kong-Macao Greater Bay Area-Taking SMN's innovative ecological product chain as an example". 


\section{REFERENCES}

André, Q., Carmon, Z., Wertenbroch, K., Crum, A., Frank, D., Goldstein, W., Huber, J., van Boven, L., Weber, B., \& Yang, H. (2018). Consumer choice and autonomy in the age of artificial intelligence and big data. Customer Needs and Solutions, 5(1), 28-37. doi:10.1007/s40547-017-0085-8

Bai, B., Guo, Z., Zhou, C., Zhang, W., \& Zhang, J. (2021). Application of adaptive reliability importance sampling-based extended domain PSO on single mode failure in reliability engineering. Information Sciences, 546, 42-59. doi:10.1016/j.ins.2020.07.069

Balthazar, P., Harri, P., Prater, A., \& Safdar, N. M. (2018). Protecting your patients' interests in the era of big data, artificial intelligence, and predictive analytics. Journal of the American College of Radiology, 15(3), 580-586. doi:10.1016/j.jacr.2017.11.035 PMID:29402532

Chen, M., Liu, Q., Huang, S., \& Dang, C. (2020). Environmental cost control system of manufacturing enterprises using artificial intelligence based on value chain of circular economy. Enterprise Information Systems, 1-20. doi:10.1080/17517575.2020.1856422

Chen, Y., \& Sivakumar, V. (2021). Invesitigation of finance industry on risk awareness model and digital economic growth. Annals of Operations Research, 1-22. doi:10.1007/s10479-021-04287-7

Cheng, S., Jianfu, S., Alrasheedi, M., Saeidi, P., Mishra, A. R., \& Rani, P. (2021). A New Extended VIKOR Approach Using q-Rung Orthopair Fuzzy Sets for Sustainable Enterprise Risk Management Assessment in Manufacturing Small and Medium-Sized Enterprises. International Journal of Fuzzy Systems, 23(5), 1-23. doi:10.1007/s40815-020-01024-3

Deng, X., Guo, X., Wu, Y. J., \& Chen, M. (2021). Perceived environmental dynamism promotes entrepreneurial team member's innovation: Explanations based on the uncertainty reduction theory. International Journal of Environmental Research and Public Health, 18(4), 2033. doi:10.3390/ijerph18042033 PMID:33669732

Dimitrov, D. V. (2019). Blockchain applications for healthcare data management. Healthcare Informatics Research, 25(1), 51-56. doi:10.4258/hir.2019.25.1.51 PMID:30788182

Ding, L., Huang, L., Li, S., Gao, H., Deng, H., Li, Y., \& Liu, G. (2020). Definition and application of variable resistance coefficient for wheeled mobile robots on deformable terrain. IEEE Transactions on Robotics, 36(3), 894-909. doi:10.1109/TRO.2020.2981822

Jiang, F., Jiang, Y., Zhi, H., Dong, Y., Li, H., Ma, S., Wang, Y., Dong, Q., Shen, H., \& Wang, Y. (2017). Artificial intelligence in healthcare: Past, present and future. Stroke and Vascular Neurology, 2(4), 230-243. doi:10.1136/ svn-2017-000101 PMID:29507784

Kibria, M. G., Nguyen, K., Villardi, G. P., Zhao, O., Ishizu, K., \& Kojima, F. (2018). Big data analytics, machine learning, and artificial intelligence in next-generation wireless networks. IEEE Access: Practical Innovations, Open Solutions, 6, 32328-32338. doi:10.1109/ACCESS.2018.2837692

Kokina, J., \& Davenport, T. H. (2017). The emergence of artificial intelligence: How automation is changing auditing. Journal of Emerging Technologies in Accounting, 14(1), 115-122. doi:10.2308/jeta-51730

Kong, H., Lu, L., Yu, J., Chen, Y., \& Tang, F. (2020). Continuous Authentication through Finger Gesture Interaction for Smart Homes Using WiFi. IEEE Transactions on Mobile Computing, 20(11), 3148-3162. doi:10.1109/TMC.2020.2994955

Kotb, A., Abdel-Kader, M., Allam, A., Halabi, H., \& Franklin, E. (2019). Information technology in the British and Irish undergraduate accounting degrees. Accounting Education, 28(5), 445-464. doi:10.1080/09639284.2 019.1588135

Liu, F., Zhang, G., \& Lu, J. (2020). Heterogeneous domain adaptation: An unsupervised approach. IEEE Transactions on Neural Networks and Learning Systems, 31(12), 5588-5602. doi:10.1109/TNNLS.2020.2973293 PMID:32149697

Liu, Z., Lang, L., Hu, B., Shi, L., Huang, B., \& Zhao, Y. (2021). Emission reduction decision of agricultural supply chain considering carbon tax and investment cooperation. Journal of Cleaner Production, 294, 126305. doi:10.1016/j.jclepro.2021.126305 
Lu, H., Li, Y., Chen, M., Kim, H., \& Serikawa, S. (2018). Brain intelligence: Go beyond artificial intelligence. Mobile Networks and Applications, 23(2), 368-375. doi:10.1007/s11036-017-0932-8

Luo, J., Meng, Q., \& Cai, Y. (2018). Analysis of the Impact of Artificial Intelligence application on the Development of Accounting Industry. Open Journal of Business and Management, 6(4), 850-856. doi:10.4236/ ojbm.2018.64063

Lv, X., \& Wu, A. (2021). The role of extraordinary sensory experiences in shaping destination brand love: An empirical study. Journal of Travel \& Tourism Marketing, 38(2), 179-193. doi:10.1080/10548408.2021.1889447

Marshall, T. E., \& Lambert, S. L. (2018). Cloud-based intelligent accounting applications: Accounting task automation using IBM watson cognitive computing. Journal of Emerging Technologies in Accounting, 15(1), 199-215. doi:10.2308/jeta-52095

Ni, T., Liu, D., Xu, Q., Huang, Z., Liang, H., \& Yan, A. (2020). Architecture of cobweb-based redundant TSV for clustered faults. IEEE Transactions on Very Large Scale Integration (VLSI) Systems, 28(7), 1736-1739. doi:10.1109/TVLSI.2020.2995094

Qiao, G., Ding, L., Zhang, L., \& Yan, H. (2021). Accessible tourism: A bibliometric review (2008-2020). Tourism Review. Advance online publication. doi:10.1108/TR-12-2020-0619

Richards, G., Yeoh, W., Chong, A. Y. L., \& Popovič, A. (2019). Business intelligence effectiveness and corporate performance management: An empirical analysis. Journal of Computer Information Systems, 59(2), 188-196. doi:10.1080/08874417.2017.1334244

Rikhardsson, P., \& Yigitbasioglu, O. (2018). Business intelligence \& analytics in management accounting research: Status and future focus. International Journal of Accounting Information Systems, 29, 37-58. doi:10.1016/j.accinf.2018.03.001

Shen, C. W., Chen, M., \& Wang, C. C. (2019). Analyzing the trend of O2O commerce by bilingual text mining on social media. Computers in Human Behavior, 101, 474-483. doi:10.1016/j.chb.2018.09.031

Shen, J., \& Han, L. (2020). Design process optimization and profit calculation module development simulation analysis of financial accounting information system based on particle swarm optimization (PSO). Information Systems and e-Business Management, 18(4), 809-822. doi:10.1007/s10257-018-00398-0

Wang, K., \& Li, S. (2021). Robust distributed modal regression for massive data. Computational Statistics \& Data Analysis, 160, 107225. doi:10.1016/j.csda.2021.107225

Wang, K., Wang, H., \& Li, S. (2021). Renewable quantile regression for streaming datasets. Knowledge-Based Systems, 107675. Advance online publication. doi:10.1016/j.knosys.2021.107675

Wu, T. J., \& Wu, Y. J. (2019). Innovative work behaviors, employee engagement, and surface acting: A delineation of supervisor-employee emotional contagion effects. Management Decision, 57(11), 3200-3216. Advance online publication. doi:10.1108/MD-02-2018-0196

Wu, W., Wang, H., \& Wu, Y. J. (2020). Internal and external networks, and incubatees' performance in dynamic environments: Entrepreneurial learning's mediating effect. The Journal of Technology Transfer, 1-27. doi:10.1007/ s10961-020-09790-w

Yan, J., Meng, Y., Yang, X., Luo, X., \& Guan, X. (2020). Privacy-Preserving Localization for Underwater Sensor Networks via Deep Reinforcement Learning. IEEE Transactions on Information Forensics and Security, 16, 1880-1895. doi:10.1109/TIFS.2020.3045320

Yang, B., \& Liao, Y. M. (2021). Research on enterprise risk knowledge graph based on multi-source data fusion. Neural Computing \& Applications, 1-14. doi:10.1007/s00521-021-05985-w PMID:34456516

Yang, R., Yu, L., Zhao, Y., Yu, H., Xu, G., Wu, Y., \& Liu, Z. (2020). Big data analytics for financial Market volatility forecast based on support vector machine. International Journal of Information Management, 50, 452-462. doi:10.1016/j.ijinfomgt.2019.05.027

Yi, H. (2021). Secure social internet of things based on post-quantum blockchain. IEEE Transactions on Network Science and Engineering, 1. Advance online publication. doi:10.1109/TNSE.2021.3095192 
Yuan, C. H., \& Wu, Y. J. (2020). Mobile instant messaging or face-to-face? Group interactions in cooperative simulations. Computers in Human Behavior, 113, 106508. doi:10.1016/j.chb.2020.106508

Zhang, M., Chen, Y., \& Susilo, W. (2020). PPO-CPQ: A privacy-preserving optimization of clinical pathway query for e-healthcare systems. IEEE Internet of Things Journal, 7(10), 10660-10672. doi:10.1109/JIOT.2020.3007518

Zhou, W., Lv, Y., Lei, J., \& Yu, L. (2019). Global and local-contrast guides content-aware fusion for RGB-D saliency prediction. IEEE Transactions on Systems, Man, and Cybernetics. Systems, 51(6), 3641-3649. doi:10.1109/TSMC.2019.2957386 